\title{
Pazopanib for the treatment of soft-tissue sarcoma
}

This article was published in the following Dove Press journal:

Clinical Pharmacology:Advances and Applications

25 October 2012

Number of times this article has been viewed

\author{
Pierre Heudel' \\ Philippe Cassier' \\ Olfa Derbel' \\ Armelle Dufresne' \\ Pierre Meeus ${ }^{2}$ \\ Philippe Thiesse ${ }^{3}$ \\ Dominique Ranchère-Vince ${ }^{4}$ \\ Jean Yves Blay' \\ Isabelle Ray-Coquard ${ }^{1,5}$ \\ 'Department of Medical Oncology, \\ ${ }^{2}$ Department of Surgical Oncology, \\ ${ }^{3}$ Department of Radiology, \\ ${ }^{4}$ Department of Pathology, Leon \\ Berard Center, Lyon, ${ }^{5}$ EAM $4 \mid 28$ \\ Sante-Individu-Societe, Lyon \\ University, Lyon, France
}

Correspondence: Isabelle Ray-Coquard Medical Oncology Department,

Center Leon Berard, 28 rue Laennec, 69008 Lyon, France

Tel +33478782888

Fax +33478782716

Email isabelle.ray-coquard@lyon.

unicancer.fr
Abstract: Pazopanib is a multikinase inhibitor which potently inhibits the activity of major receptor tyrosine kinases, including vascular endothelial growth factor receptor-1, vascular endothelial growth factor receptor-2, vascular endothelial growth factor receptor-3, plateletderived growth factor receptor-a, platelet-derived growth factor receptor-a, and c-Kit. Approved by the Food and Drug Administration in 2009 in the United States for the treatment of metastatic renal cell carcinoma, pazopanib has been tested in advanced or metastatic soft-tissue sarcoma. Unlike other tyrosine kinase inhibitors, a statistically significant efficacy in phase II but also in randomized phase III studies has been shown. In comparison with sunitinib or sorafenib, pazopanib has a similar toxicity profile and is generally well tolerated. This review details the development of this new therapeutic class in the treatment of metastatic soft-tissue sarcomas.

Keywords: soft-tissue sarcoma, pazopanib, tyrosine kinase inhibitor

\section{Introduction}

Soft-tissue sarcomas (STS) comprise a heterogeneous group of neoplasms originating from mesenchymal tissues. These tumors can arise in any area of the body, but develop predominantly in the limbs and abdominal cavity. They account for $<1 \%$ of all cancers and have more than 50 histological types exhibiting major differences in terms of pathogenesis, genetic alterations, and clinical behavior.

Despite major progress in the classification of sarcomas, the treatment of these rare tumors has remained surprisingly homogeneous both in localized and advanced settings. Indeed, in a localized setting, primary biopsy followed by en bloc resection of the tumor and adjuvant radiotherapy represent the standard therapeutic approach for tumors $>5 \mathrm{~cm}$, deeply seated, and FNCLCC (Federation Nationale des Centres de Lutte Contre le Cancer) grade $>1$. Chemotherapy is based on doxorubicin and ifosfamide, and can be delivered as a neoadjuvant treatment in locally advanced tumors to shrink the tumor and subsequently enable removal of the tumor. ${ }^{1}$ In an advanced setting, doxorubicin, ifosfamide, dacarbazine, and trabectedin remain the only registered cytotoxics for this indication, while imatinib - in gastrointestinal stromal tumors and sunitinib - in second-line gastrointestinal stromal tumors - are the only registered agents. Until recently, the homogeneity of clinical management contrasted sharply with the heterogeneity of disease types, which were being extensively characterized at the molecular level. ${ }^{2}$

Prognosis in the latter setting is poor, with a median overall survival and 5-year overall survival rate of approximately 12 months and $8 \%$, respectively, although 
improvement over the years has been noted. ${ }^{3}$ Literature data illustrate the demand for more active treatments. One of these approaches is to target angiogenesis in sarcoma tumor progression and metastasis. Tumor vascular endothelial growth factor (VEGF) expression correlates with histologic grade, stage, and clinical outcome. ${ }^{4} \mathrm{~A}$ treatment that is sufficient to inhibit tumor angiogenesis could thus be interesting for treatment in STS. Other multikinase inhibitors (eg, sunitinib or sorafenib) have been tested in patients with advanced STS, but generally with limited success in phase II trials and with only minor activity in certain STS subtypes. ${ }^{5,6}$ Pazopanib, an orally available agent targeting VEGF receptors, plateletderived growth factor (PDGF) receptors, and c-Kit, seems to be a promising agent. Compared with historical controls receiving second-line chemotherapy, pazopanib extended progression-free survival and overall survival in patients with STS. In this article, the clinical development and emerging role of pazopanib in the treatment of advanced STS is discussed.

\section{Preclinical data}

In vitro studies showed that pazopanib is a multitargeted, tyrosine kinase inhibitor with activity against VEGF receptor-1 (VEGFR1), VEGFR2, VEGFR3, and PDGF.' In vivo, pazopanib demonstrated tumor growth inhibition significantly dependent on the administered dose for a wide variety of tumor xenografts (especially renal cell cancer) in immunocompromised mice. ${ }^{8}$ Its effect on VEGF-induced VEGFR2 phosphorylation was evaluated in mice by administering an intravenous bolus of VEGF. Mice lungs receiving an intravenous bolus of VEGF had a larger phosphorylation of VEGFR2 than untreated control mice. When mice previously received a single dose of pazopanib, inhibition of VEGF-induced VEGFR2 phosphorylation in lungs was dose- and time-dependent. A single oral dose of $30 \mathrm{mg} / \mathrm{kg}$ pazopanib inhibited phosphorylation for more than 8 hours. In vivo, these results showed that the optimal inhibition of VEGFR2 phosphorylation requires plasma concentrations higher than $40 \mu \mathrm{mol} / \mathrm{L} .{ }^{9}$

In comparison to sorafenib or sunitinib, the pharmacokinetic characteristics of pazopanib are similar, and, like other tyrosine kinase inhibitors, pazopanib is highly proteinbound. It is metabolized by cytochrome 3A4 (CYP3A4), but interactions with CYP3A4 inducers or inhibitors have not been described yet. ${ }^{10}$ However, coadministration of strong CYP3A4 inhibitors (eg, clarithromycin or ketoconazole), grapefruit juice, and CYP3A4 inducers (eg, rifampin) should be avoided. Pazopanib, sunitinib, and sorafenib are hepatically metabolized and eliminated via the bowels. Renal elimination is low. Pazopanib has a significantly lower percentage of renal elimination than sorafenib and sunitinib. ${ }^{11}$

\section{Phase I}

A phase I study reported by Hurwitz et al evaluated a variety of doses and schedules of pazopanib (50 mg and $100 \mathrm{mg}$ three times a week, 50-2000 mg once a day, and $300 \mathrm{mg}$ and $400 \mathrm{mg}$ twice a day) in 63 patients with advanced-stage and refractory solid tumors. ${ }^{10}$ Although a maximum tolerated dose was not obtained in this phase I trial, absorption at doses above $800 \mathrm{mg}$ once a day is limited because the oral bioavailability of pazopanib is low. Therefore, the 800-mg daily dose group was selected for use in phase II and III clinical trials. Pazopanib is administered between meals (either 1 hour before or 2 hours after eating) because its absorption may increase when administered with food.

Seventeen patients had significant clinical benefit with pazopanib and 14 patients had stable disease for more than 6 months. Four patients with sarcoma (one with leiomyosarcoma, two with chondrosarcoma, and one with gastrointestinal stromal tumor) achieved prolonged stable disease for at least 6 months. The most frequently reported side effects of all grades were hypertension (33\%) easily controlled with antihypertensive medication, diarrhea (33\%), nausea $(32 \%)$, and hair depigmentation (32\%). It should be noted that there were single events of gastrointestinal bleeding and deep vein thrombosis with pulmonary embolism.

\section{Phase II}

In a phase II trial, pazopanib was tested in 142 patients with relapsed or refractory advanced STS. ${ }^{12}$ Pazopanib was dosed at $800 \mathrm{mg}$ once a day. Four strata were studied: liposarcomas, leiomyosarcomas, synovial sarcomas, and other STS types. The median follow-up for efficacy was 677 days. The liposarcoma stratum was discontinued for 17 first-stage patients who did not reach the prespecified primary endpoint of $20 \%$ progression-free rate at 12 weeks. Progression-free rate at 12 weeks was $44 \%$ in the leiomyosarcoma cohort, $49 \%$ in the synovial sarcoma cohort, and 39\% in the other STS subtype cohort. Response rate, median progression-free survival, and median overall survival are summarized in Table 1.

In comparison with historical controls of soft-tissue sarcomas treated with second-line chemotherapy, progressionfree survival and overall survival were prolonged in the three strata (leiomyosarcomas, synovial sarcomas, other STS subtypes). ${ }^{13}$ Additionally, some patients with RECIST (Response Evaluation Criteria in Solid Tumors) stable 
Table I Results of phase II trial

\begin{tabular}{llll}
\hline Strata & Response rate & Progression-free survival & Overall survival \\
\hline Liposarcomas $(n=19)$ & $0 \%$ & 2.9 months & 6.6 months \\
Leiomyosarcomas $(n=42)$ & $2.4 \%$ & 3.3 months & 11.8 months \\
Synovial sarcomas $(n=38)$ & $13.2 \%$ & 5.8 months & 10.3 months \\
Other sarcomas $(n=43)$ & $7 \%$ & 3.3 months & 9.9 months \\
\hline
\end{tabular}

disease criteria presented cystic alterations in major target lesions, suggesting drug response.

The most common side effects were anemia (74.6\%), leukopenia (42.2\%), liver enzyme elevations (50\%), and proteinuria $(51.4 \%)$. The most frequent adverse events considered to be drug-related included hypertension, fatigue, hair depigmentation, and nausea. The most frequent grade $3-4$ side effects were hypertension (7.7\%), fatigue (7.7\%), and hyperbilirubinemia $(6.3 \%) ; 60 \%$ of patients required treatment interruptions, and $23 \%$ patients required dose reductions. Nonhematologic toxicities were the main cause of interruptions and reductions. A randomized phase III trial was delineated further to these encouraging results in term of safety and efficacy.

\section{Phase III}

PALETTE (Pazopanib Explored In STS) study is an international multicenter (EORTC 62072), double-blind, placebocontrolled phase III trial. This trial compared pazopanib versus placebo, with 2:1 randomization and no crossover, after at least two metastatic lines of chemotherapy. Patients were randomized to receive either placebo $(n=123)$ or pazopanib $800 \mathrm{mg}$ once a day $(\mathrm{n}=246)$ until tumor progression, unacceptable toxicity, death, or at the patient's request. Median age was 56 years and median duration of follow-up at clinical cutoff date was 15 months. The primary endpoint was progression-free survival (by independent central review). The results were presented at the 2011 annual meeting of the American Society of Clinical Oncology and published recently. ${ }^{14} \mathrm{~A}$ total of 369 patients in 13 countries were included between October 2008 and February 2010. Patients should not have previously received more than four monotherapies or two polychemotherapies, and were pretreated by antracyclines. Pazopanib was given after two lines of treatment, but not specifically for maintenance therapy.
The study showed $69 \%$ reduction in the risk of progression or death in patients who received pazopanib. Patients receiving pazopanib had a higher progression-free survival than those receiving placebo (4.6 months versus 1.6 months, respectively; hazard ratio $=0.31 ; P<0.0001)$. However, the difference in overall survival was not statistically significant with 12.5 months in the pazopanib arm versus 10.7 months in the placebo arm $(P=0.25$; hazard ratio $=0.86$, data of the final analysis). Response rate, median progression-free survival, and median overall survival are summarized in Table 2.

Main on-therapy grade 3-4 toxicities in the pazopanib versus placebo arm, respectively, were fatigue ( $13 \%$ versus $6 \%$ ), hypertension ( $7 \%$ versus $0 \%$ ), anorexia ( $6 \%$ versus $0 \%$ ), and diarrhea (5\% versus $1 \%$ ). Similarly, grade $3-5$ thromboembolic events ( $3 \%$ versus $2 \%$ ) and left ventricular ejection fraction drop of $>15 \%$ ( $8 \%$ versus $3 \%)$ were experienced in the pazopanib versus placebo arm, respectively.

\section{Combination with other targeted therapies or chemotherapy}

No combination with other targeting therapeutic agents has been performed in the treatment of STS. Nevertheless, a phase II trial of pazopanib or lapatinib compared with pazopanib plus lapatinib combination therapy was performed on patients with advanced cervical cancer. However, at the planned intermediate analysis, toxicity was higher in the combination therapy compared to pazopanib or lapatinib monotherapy and efficacy was similar. ${ }^{15}$ The results of a French phase I study were presented at the 2012 annual meeting of the American Society of Clinical Oncology. This trial explored the safety and feasibility of the combination of pazopanib with bevacizumab in 15 patients with advanced refractory solid tumors (seven patients with renal cell carcinoma, but no patients with sarcoma). In nephrectomized patients, the maximal therapeutic dose of pazopanib was $400 \mathrm{mg}$ a day,

Table 2 Results of phase III trial

\begin{tabular}{lllll}
\hline & Objective response & Stable disease & Progression-free survival & Overall survival \\
\hline Pazopanib $(n=246)$ & $9 \%$ & $67 \%$ & 4.6 months & 12.5 months \\
Placebo $(n=123)$ & $0 \%$ & $38 \%$ & 1.6 months & 10.7 months \\
\hline
\end{tabular}


and has not been reached yet in other patients. ${ }^{16}$ Furthermore, in patients with advanced gynecologic tumors, a phase I/II study planned to evaluate the safety and efficacy of paclitaxel plus carboplatin once every 3 weeks for up to six cycles with either $800 \mathrm{mg}$ or $400 \mathrm{mg}$ per day pazopanib. Severe myelotoxicity was reported in six of the twelve patients and two gastrointestinal perforations. In conclusion, combining pazopanib with carboplatin/paclitaxel chemotherapy is not a feasible treatment. ${ }^{17}$

\section{Toxicity profile}

In comparison with other tyrosine kinase inhibitors (eg, sorafenib or sunitinib), pazopanib showed similar toxicity profiles in major published clinical trials performed in metastatic renal cell carcinoma. Some side effects may be associated to a class effect such as diarrhea, fatigue, skin effects (rash, hair depigmentation, hand-foot syndrome), and transaminase elevation. Due to its antiangiogenic activities, pazopanib has been related with hypertension, bleeding, venous and arterial thrombosis, and proteinuria. The most frequent grade 3-4 side effects are summarized in Table 3.

Of note, some lethal hepatitis has been described with pazopanib, and elevations in transaminases and total bilirubin must be closely monitored. Patients with Gilbert's syndrome must be monitored because pazopanib inhibits UDP-glycosyltransferase-1 family, polypeptide A1, an enzyme involved in the metabolism of bilirubin and hyperbilirubinemia induced by pazopanib that has been associated with a polymorphism of the gene for UDP-glycosyltransferase-1 family, polypeptide A1 found in patients with Gilbert's syndrome. ${ }^{18}$

A left ventricular ejection fraction drop has also been described with pazopanib. In the PALETTE (Pazopanib Explored In STS) study, a left ventricular ejection fraction drop of more than $15 \%$ occurred in 21 patients $(8 \%)$ in the pazopanib group during or after treatment; however, left ventricular ejection fraction later improved in most cases. ${ }^{14}$

Table 3 Most frequent grade 3-4 adverse events

\begin{tabular}{llll}
\hline $\begin{array}{l}\text { Most frequent } \\
\text { grade 3-4 adverse events }\end{array}$ & $\begin{array}{l}\text { Phase I } \\
\text { trial (\%) }\end{array}$ & $\begin{array}{l}\text { Phase II } \\
\text { trial (\%) }\end{array}$ & $\begin{array}{l}\text { Phase III } \\
\text { trial (\%) }\end{array}$ \\
\hline Hypertension & 25 & 7.7 & 7 \\
Fatigue & 2 & 7.7 & 13 \\
Anorexia & 0 & - & 6 \\
Diarrhea & 5 & 3.5 & 5 \\
Thromboembolic event & 6 & 0.007 & 3 \\
Hyperbilirubinemia & 2 & 6.3 & - \\
Elevated transaminases & 2 & 4.2 & - \\
Proteinuria & 3 & 0 & - \\
\hline
\end{tabular}

\section{Discussion}

Despite heterogeneity and variability of STS histological subtypes, the treatment of these rare tumors has remained remarkably homogeneous, and systemic chemotherapy including agents such as doxorubicin or ifosfamide remains, for the moment, the benchmark. Important progress has been made in the understanding of molecular alterations, which, associated with sarcoma subtypes, enabled the identification of early mutational events during tumor progression such as translocations and mutation of tyrosine kinases. ${ }^{19}$ Growth factors and their corresponding receptors (VEGF and VEGFR for example) or molecules important in intracellular signaling pathways (eg, mammalian target of rapamycin) and molecules with involvement in neoplastic evolution are interesting targets for new treatment. Multikinase inhibitors have been tested in patients with advanced STS, generally with limited success reported to date. Sunitinib (37.5 mg/day continuous daily dosing $)$ in a phase II $(\mathrm{n}=28)$ study produced one partial response and stable disease in ten patients. ${ }^{6}$ In another phase II study, Mahmood et al treated 48 patients with sunitinib $50 \mathrm{mg}$ /day on a 4-week on/2-week off schedule. In this study, only one partial response was seen and the 3-month progression-free survival rate was $58 \%{ }^{20}$ Maki et al reported a phase II study with sorafenib enrolling 147 patients with various subtypes of STS. The 3-month progression-free survival rate for the whole population was $53 \%{ }^{5}$

Pazopanib is a second-generation tyrosine kinase inhibitor with highly selective activity against VEGFR, PDGFR, and c-KIT, which has demonstrated significant clinical benefit in a variety of malignancies, especially for the treatment of metastatic renal cell carcinoma. ${ }^{21}$ In advanced or metastatic STS, a previous phase I trial suggested significant activity of pazopanib and was confirmed in a large phase II study. The PALETTE (Pazopanib Explored In STS) study was the first randomized phase III trial demonstrating the efficacy of an antiangiogenic agent in pretreated STS. ${ }^{22}$ In this phase III trial, pazopanib was given after two lines of treatment, but not specifically for maintenance therapy. Maintenance therapy has been developed as part of treatment paradigms to prolong response duration and delay disease progression in responsive patients or patients with stable disease after a defined number of chemotherapy cycles. ${ }^{23}$ Given the inherent toxicity associated with first-line chemotherapy and the need to carry on maintenance therapy for prolonged periods of time, agents used in maintenance therapy should be well tolerated. The SUCCEED (Sarcoma Multicenter Clinical Evaluation of the Efficacy of Ridaforolimus) trial is a pivotal phase III trial that evaluated maintenance therapy with oral ridaforolimus, 
a mammalian target of rapamycin inhibitor, in patients with advanced sarcoma $(n=711) \cdot{ }^{24}$ A progression-free survival benefit was observed in patients receiving ridaforolimus compared to placebo (17.7 months versus 14.6 months, respectively). In terms of overall survival, the benefit for ridaforolimus-treated patients was small compared to patients receiving placebo. Considering these results and due to the specific VEGF pathway inhibited by pazopanib, more physicians will be interested in the molecular mechanism of this drug compared to mammalian target of rapamycin inhibitors. Effectively, it is more important - for patients with metastatic disease - to maintain quality of life and control the tumor burden with manageable drug toxicity for as long as possible than to obtain an objective response.

The emergence of molecularly targeted therapies over the last decade has led to rationally based, personalized management for patients with advanced or metastatic disease based on current understanding of specific sarcoma histiotype biology. ${ }^{25}$ Nevertheless, much remains to be determined. Future strategies for these agents will have to identify patients who will benefit from these agents and which histology could be treated with each targeted therapy. It will be also necessary to validate surrogate markers to test the efficacy of the drug on the target and associated therapies to yield improved outcomes by controlling different mechanisms of resistance.

\section{Conclusion}

In view of these results, the therapeutic possibilities for patients with advanced or metastatic STS will certainly change in the coming years. Treatment will be more individualized, probably with a better determination of molecular targets, which will require a better understanding into the molecular biology of STS histological subtypes. From results of a phase III trial, pazopanib is the first drug targeting angiogenesis which has shown activity in antracycline-pretreated advanced STS.

\section{Disclosure}

The authors report no conflicts of interest in this work.

\section{References}

1. Casali PG, Blay JY. Soft tissue sarcomas: ESMO Clinical Practice Guidelines for diagnosis, treatment and follow-up. Ann Oncol. 2010; 21(Suppl 5):v198-v203.

2. Blay JY, Cassier PA, Ray-Coquard I. Soft tissue sarcomas: are all soft tissue sarcomas treated with the same drugs? Eur J Cancer. 2011;47(Suppl 3): S385-S388.

3. Italiano A, Mathoulin-Pelissier S, Cesne AL, et al. Trends in survival for patients with metastatic soft-tissue sarcoma. Cancer. 2011;117(5): $1049-1054$.
4. DuBois S, Demetri G. Markers of angiogenesis and clinical features in patients with sarcoma. Cancer. 2007;109(5):813-819.

5. Maki RG, D'Adamo DR, Keohan ML, et al. Phase II study of sorafenib in patients with metastatic or recurrent sarcomas. J Clin Oncol. 2009;27(19):3133-3140.

6. George S, Merriam P, Maki RG, et al. Multicenter phase II trial of sunitinib in the treatment of nongastrointestinal stromal tumor sarcomas. J Clin Oncol. 2009;27(19):3154-3160.

7. Harris PA, Boloor A, Cheung M, et al. Discovery of 5-([4-[[2, 3- dimethyl-2H-indazol-6-yl]methylamino]-2-pyrimidinyl]amino)2-methyl-benzenesulfonamide (Pazopanib), a novel and potent vascular endothelial growth factor receptor inhibitor. $J$ Med Chem. 2008;51(15):4632-4640.

8. Kumar R, Knick VB, Rudolph SK, et al. Pharmacokinetic-pharmacodynamic correlation from mouse to human with pazopanib, a multikinase angiogenesis inhibitor with potent antitumor and antiangiogenic activity. Mol Cancer Ther. 2007;6(7):2012-2021.

9. Bukowski RM. Pazopanib: a multikinase inhibitor with activity in advanced renal cell carcinoma. Expert Rev Anticancer Ther. 2010;10(5):635-645.

10. Hurwitz HI, Dowlati A, Saini S, et al. Phase I trial of pazopanib in patients with advanced cancer. Clin Cancer Res. 2009;15(12): $4220-4227$.

11. Keisner SV, Shah SR. Pazopanib: the newest tyrosine kinase inhibitor for the treatment of advanced or metastatic renal cell carcinoma. Drugs. 2011;71(4):443-454.

12. Sleijfer S, Ray-Coquard I, Papai Z, et al. Pazopanib, a multikinase angiogenesis inhibitor, in patients with relapsed or refractory advanced soft tissue sarcoma: a phase II study from the European organisation for research and treatment of cancer-soft tissue and bone sarcoma group (EORTC study 62043). J Clin Oncol. 2009;27(19): 3126-3132.

13. Van Glabbeke M, Verweij J, Judson J, Nielsen OS. Progression-free rate as the principal end-point for Phase II trials in soft-tissue sarcomas. Eur J Cancer. 2002;38(4):543-549.

14. van der Graaf WT, Blay J, Chawla SP, et al. Pazopanib for metastatic soft-tissue sarcoma (PALETTE): a randomised, double-blind, placebocontrolled phase 3 trial. Lancet. 2012;379(9829):1879-1886.

15. Monk BJ, Mas Lopez L, Zarba JJ, et al. Phase II, open-label study of pazopanib or lapatinib monotherapy compared with pazopanib plus lapatinib combination therapy in patients with advanced and recurrent cervical cancer. J Clin Oncol. 2010;28(22):3562-3569.

16. Negrier S, Perol D, Bahleda R, et al. A phase I study of pazopanib (P) combined with bevacizumab (B) in patients with metastatic renal cell carcinoma (mRCC) or other advanced refractory tumors [abstract]. J Clin Oncol. 2012;30:4614.

17. du Bois A, Vergote I, Wimberger P, et al . Open-label feasibility study of pazopanib, carboplatin, and paclitaxel in women with newly diagnosed, untreated, gynaecologic tumours: a phase I/II trial of the AGO study group. Br J Cancer. 2012;106(4):629-632.

18. Xu CF, Reck BH, Xue Z, et al. Pazopanib-induced hyperbilirubinemia is associated with Gilbert's syndrome UGT1A1 polymorphism. $\mathrm{Br} J$ Cancer. 2010;102(9):1371-1377.

19. Clark MA, Fisher C, Judson I, Thomas JM. Soft-tissue sarcomas in adults. N Engl J Med. 2005;353(7):701-711.

20. Mahmood ST, Agresta S, Vigil C, et al. Phase II study of sunitinib malate, a multi-targeted tyrosine kinase inhibitor in patients with relapsed or refractory soft tissue sarcomas. Focus on 3 prevalent histologies: leiomyosarcoma, liposarcoma, and malignant fibrous histiocytoma. Int $J$ Cancer. December 10, 2010. [Epub ahead of print.]

21. Sternberg CN, Szczylik C, Lee E, et al. A randomized, double-blind phase III study of pazopanib in treatment-naive and cytokine-pretreated patients with advanced renal cell carcinoma (RCC) [abstract]. J Clin Oncol. 2009;27(Suppl 15):5021.

22. Kasper B, Hohenberger P. Pazopanib: a promising new agent in the treatment of soft tissue sarcomas. Future Oncol. 2011;7(12): 1373-1383. 
23. Ray-Coquard I. Maintenance therapy in the treatment of sarcoma. Clin Adv Hematol Oncol. 2011;9(10):773-774.

24. Chawla SP, Blay J, Ray-Coquard IL, et al. Results of the phase III, placebo-controlled trial (SUCCEED) evaluating the mTOR inhibitor ridaforolimus $(\mathrm{R})$ as maintenance therapy in advanced sarcoma patients (pts) following clinical benefit from prior standard cytotoxic chemotherapy (CT) [abstract]. J Clin Oncol. 2011;29:10005.
25. Cassier PA, Labidi-Galy SI, Heudel P, et al. Therapeutic pipeline for softtissue sarcoma. Expert Opin Pharmacother. 2011;12(16):2479-2491.

\section{Publish your work in this journal}

Clinical Pharmacology: Advances and Applications is an international, peer-reviewed, open access journal publishing original research, reports,

reviews and commentaries on all areas of drug experience in humans.

The manuscript management system is completely online and includes

a very quick and fair peer-review system, which is all easy to use.
Visit http://www.dovepress.com/testimonials.php to read real quotes from published authors. 